\title{
Risk factors associated with nonvaccination rabies status of dogs in KwaZulu-Natal, South Africa
}

\section{Melinda Hergert ${ }^{\prime}$ \\ Kevin le Roux ${ }^{2}$ \\ Louis $\mathrm{H} \mathrm{Nel}^{3,4}$}

'Department of Paraclinical Sciences, Faculty of Veterinary Science, University of Pretoria, Onderstepoort, Pretoria, ${ }^{2} \mathrm{KwaZulu}$-Natal Department of Environment, Agriculture and Rural Development, Government Veterinary Services, Pietermaritzburg, ${ }^{3}$ Department of Microbiology and Plant Pathology, Faculty of Natural and Agricultural Sciences, University of Pretoria, Pretoria, South Africa; ${ }^{4}$ Global Alliance for Rabies Control, Manhattan, KS, USA
Correspondence: Melinda Hergert Department of Paraclinical Sciences, Faculty of Veterinary Science, University of Pretoria, Private Bag X04, Onderstepoort 0I I0, South Africa Email melindahergert@yahoo.com
This article was published in the following Dove Press journal:

Veterinary Medicine: Research and Reports

27 June 2016

Number of times this article has been viewed

\begin{abstract}
Canine rabies has been enzootic in the dog population of the KwaZulu-Natal province of South Africa since the mid-1970s and has been associated with high rates of human exposures and frequent transmissions to other domestic animal species. Several decades of control efforts, consisting primarily of mass vaccination programs, failed to sufficiently curb rabies in this province. For meaningful progression toward better control and elimination, the factors contributing to the persistence of this disease need to be elucidated and addressed. This paper reports evaluated observations from survey records captured through a cross-sectional observational study regarding owned canine populations in this South African province. We used logistic regression modeling to predict variables associated with risk of nonvaccination of rabies in owned dogs. The study indicated that husbandry practices, rabies knowledge, geographical area/location, and the ages of dogs were important factors associated with the risk of nonvaccination. High population turnover, together with large free roaming dog populations, compromised the levels of vaccination achieved and contributed to the persistence of dog rabies in the province. Dog owners in this study also reported that they were more likely to present their dogs for vaccination when the vaccines were free of charge (52\%) and less than a kilometer from their homes (91\%). It has been suggested that effective dog rabies control requires $70 \%$ or more of the dog population to be vaccinated. Our data showed that this figure was not reached in the surveyed dog population.
\end{abstract}

Keywords: dog population, canine rabies, rabies vaccination, vaccination campaigns, KwaZulu-Natal, South Africa, dog owners

\section{Introduction}

The World Health Organization recommends that at least $70 \%$ of the dog population should be vaccinated in order to prevent or control a dog rabies outbreak. ${ }^{1}$ Results from rabies disease transmission modeling concur that the vaccination of $70 \%$ of dogs would effectively control dog rabies and prevent over $90 \%$ of human rabies cases. ${ }^{2}$ To this end, accessibility to dogs is one of the most important factors in successful rabies vaccination campaigns. ${ }^{1}$ It has been suggested that campaigns should be conducted during hours when owners will be home to present and help handle their dogs particularly in working class communities or during school holidays as many workers have found that school aged boys frequently present the dogs for vaccination. ${ }^{3,4}$ In some cultural settings, a central point vaccination station can be established, such as has been carried out in the agro-pastoral communities in Tanzania. ${ }^{5}$ However, in KwaZulu-Natal $(\mathrm{KZN})$, South Africa, and pastoral Tanzania where rural housing is more dispersed, 
it has been proven that dog owners will not travel far, and the door-to-door approach to dog vaccination is more effective. ${ }^{5,6}$ Disease awareness, religion, and culture can affect individual participation in the form of dog accessibility through owner presentation and desire for rabies vaccination as seen in Muslim communities of Ethiopia and Maasai communities in northwestern Tanzania. ${ }^{7,8}$ The purpose and source of the dog can affect vaccination status - for instance, dogs obtained inexpensively or for free were more at risk of nonvaccination in both Mexico and Nigeria.. ${ }^{9,10}$ Related dog management factors can be considered together under the general definition of animal husbandry. Uncontrolled or free-roaming dogs can be associated with nonvaccination. ${ }^{11}$ In Chad, dog owners were less likely to present their dogs for rabies vaccine as the price for vaccine rose above US\$ $1 .^{12}$ The Eastern Cape province of South Africa reported that free vaccine delivery and location were both factors in achieving adequate vaccination coverage. ${ }^{13}$ Many canine ecology studies have indicated that the age of the dog is relevant in the risk of nonvaccination. ${ }^{9,14,15}$

Canine rabies was introduced to the KZN province of South Africa in the 1960s from Mozambique. The disease has been maintained in the domestic dog population despite a considerable control effort that includes free of charge, annual mass vaccination campaigns. During campaigns, owners receive a card with the date, number of animals of each species vaccinated, owner surname, and signature of the animal health technician administering the vaccine. These cards help identify that animals in a household were vaccinated by government veterinary services (GVS), but is less ideal than a detailed vaccination certificate, which would ascertain vaccination of individual animals. ${ }^{4}$ For logistical reasons, the issuing of comprehensive vaccination certificates or collars during mass campaigns has been found to be impractical and expensive by KZN-GVS.

Frequent transmissions of rabies from dogs to humans and other domestic animal species have been commonplace over the past few decades. ${ }^{4,16}$ The factors contributing to the persistence of canine rabies in the face of several decades of control efforts and mass vaccination programs are likely to be multifaceted. Clearly, the ideal vaccination target is not reached, and the reasons for this need to be elucidated and addressed.

The purpose of this study was to estimate the vaccination coverage of the owned dog population in surveyed areas using owner reported data captured as part of a larger dog population study. Logistic regression modeling is used to predict variables associated with the risk of nonvaccination of the owned canine metapopulation.

\section{Methods}

\section{Study area and sampling procedure}

From September 2009 through January 2011, household surveys were conducted in six different communities across the KZN province, covering three land use types: rural, urban, and peri-urban (Figure 1). Affluent urban and suburban areas where people keep dogs in confined spaces have lower rabies risk due to fewer affective contacts between animals and easier access to veterinary services. ${ }^{17}$ Such areas were therefore excluded in our study. Poorer urban townships and rural villages represent the areas from where canine rabies is most frequently reported by KZN Department of Agriculture, Environment and Rural Development and the effective study areas were selected with the assistance of the KZN Department of Agriculture, Environment and Rural Development GVS division. From a total of 1,992 participating households, the community distribution was 52\% rural, 33\% urban, and $15 \%$ peri-urban. The urban areas were high density townships consisting of formal and informal housing with tarred roads, running water, and no livestock. Rural areas consisted of rectangular block houses or traditional Zulu style rondavels (round southern Africa native huts usually made of mud), dirt roads, no running water, and $>27 \%$ livestock owning households. The peri-urban area contained formal housing, tarred roads, $87 \%$ running water, and $2 \%$ livestock owning households. Rabies was enzootic in all areas, with the exception of the peri-urban community of Wembezi.

Simple random sampling and systematic surveys are difficult in developing countries due to logistical and sometimes adverse cultural or political reasons. ${ }^{15}$ Therefore, random sampling using a cluster or "area" design was used because homesteads in rural areas are not numbered and informal housing settlements within townships are frequently arranged haphazardly. ${ }^{18}$ Clusters for this survey were developed using Google Earth maps at $4.6 \mathrm{~km}$ eye altitude with a grid in order to maintain a consistent sampling methodology. Reliable aerial photographs of each community were not available. Consistent use of maps permits the random systematic selection of similar sampling units between differing geographical areas. ${ }^{1}$ Each block of the grid represented a cluster and was numbered from left to right. Clusters were selected for sampling with replacement by entering numbers into an online random number generator in order to select blocks of households within the community to be surveyed. ${ }^{19}$ The desired 


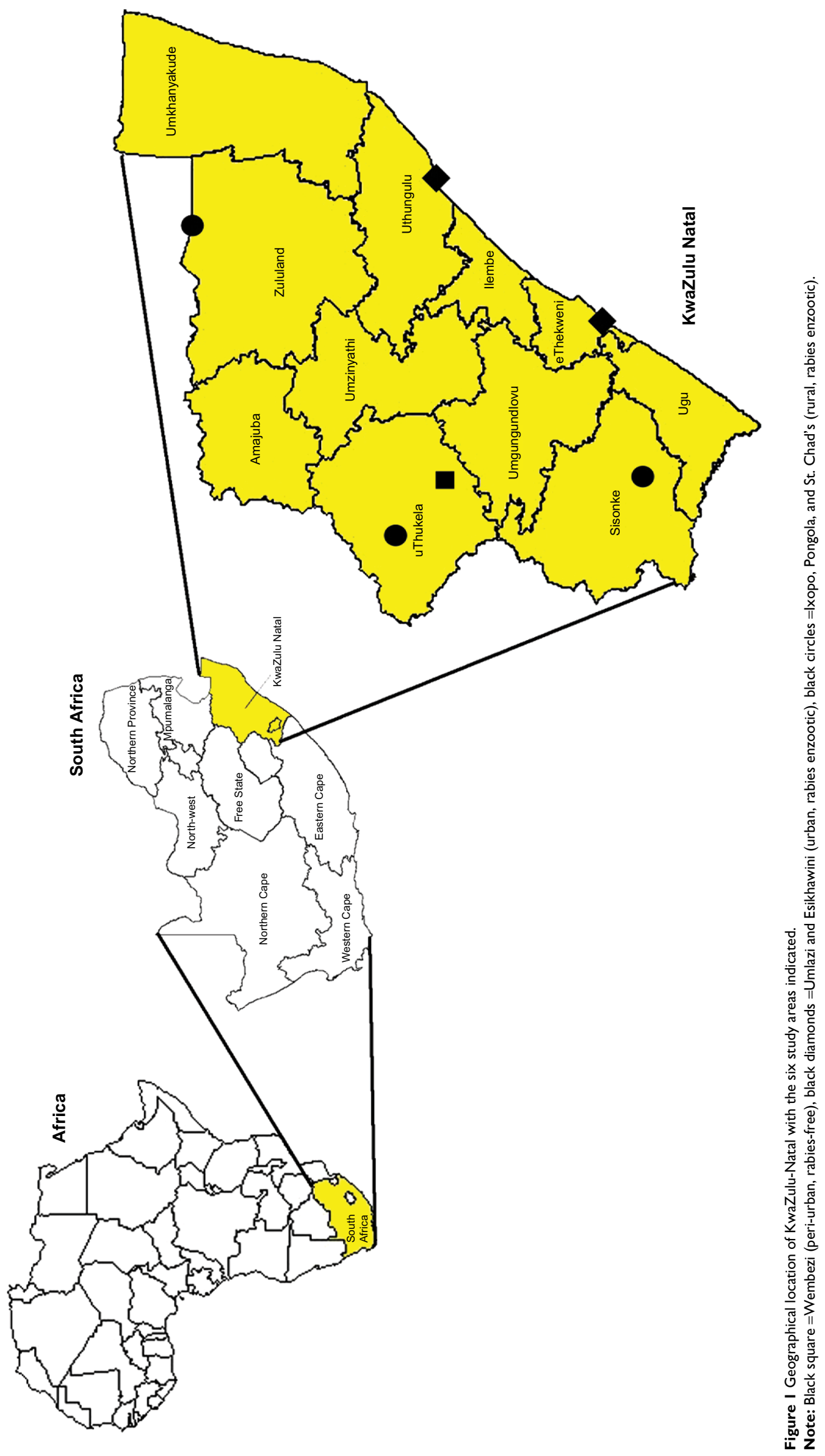


minimum sample size for each area was 323 households. In the urban townships, few blocks were selected due to the high concentration of houses in each block whereas in rural areas $>90 \%$ of blocks were sampled because of the low housing density and relative human population. All houses within a selected block were sampled regardless of community type. There is potential for extrapolating survey results into the entire study area permitting generalizations provided that the geographical, socioeconomic, and culture settings are the same or very close. ${ }^{1}$

\section{Questionnaire interviews}

Based upon the World Health Organization dog rabies control guidelines, the questionnaires were composed of two parts: a survey for collecting household demographics regarding the residents and a survey for individual descriptive statistics of each owned dog in the population. ${ }^{20}$ The surveys were translated into isiZulu and then back translated to English before being piloted in a township with a history of canine rabies. KZN Department of Agriculture, Environment and Rural Development animal health technicians and students, Department of Health workers, Environmental Health workers, and SPCA employees were trained to perform the surveys. All interviews were conducted between the hours of 9 am and 3 pm.

\section{Data analysis}

The data from each area were entered into a Microsoft Excel spreadsheet and imported into SAS Version 9.3 (SAS Institute, Inc., Cary, NC, USA). The unit of observation for this particular study was risk of nonvaccination at the dog level. Initial screening of potential risk factors for nonvaccination was performed with univariable chi-square test. The level of significance necessary to enter a starting model was $P \leq 0.05$. When all of the significant variables were entered into the effects model separately, there were problems with convergence and evidence of interactions of some related variables. Further analysis to identify variables with relationships was performed. Three constructs were made from logically interrelated predictor variables that could collectively describe characteristics about the dog, the household in which it lived, and the husbandry practices used in caring for the dog. The new variable for describing the dog was constructed by combining the dog's source and purpose. The new husbandry variable was constructed from the pool of variables describing provision of shelter, how much of the day the dog was chained, if it wore a collar, what food it was fed, and who could handle the dog. The household variable was created by combining ownership of livestock, the number of dogs in the household (1-3 or $>3$ ), and if the respondent had knowledge of rabies. The number of dogs per household was included in the model under the household construct in order to cope with the fact that some of the household data would be repeated in the model in describing factors at the dog level. The three new constructs were ranked on a scale of low, medium, and high as a scoring system. Sex of the dog was insignificant in univariate testing. The age of the dog was considered to be an important explanatory variable and was entered separately. We ran a stepwise logistic regression model to predict rabies vaccination as "yes or no". ${ }^{21}$

\section{Ethics statement}

The study was approved by the University of Pretoria Veterinary Faculty Research Committee and non-experimental use of animals by the Animal Use and Care Committee. Prior to the interview, the purpose of the survey was explained to each participant in their native language at which point they could either accept and provide written informed consent or decline to participate.

\section{Results \\ Household characteristics}

The response rates for the door-to-door interviews ranged from $92 \%$ to $100 \%$ in the six areas surveyed. A total of 1,992 households consisting of 13,756 people (range 1-34, median $=6$ ) completed the surveys within the three community types. Surveys were answered by a person defined as head of the household in $68 \%(1,361 / 1,992)$ of the cases across the province (range $63 \%-76 \%$ ). The remainder of respondents were children accompanied by an adult relative $(1 \%, n=11)$, children aged 14 and older $(9 \%, n=183)$, or an adult other than the head of the household $(22 \%, n=435)$.

Ninety-eight percent $(1,949 / 1,992)$ of the population was of Zulu culture. Seventy-nine percent $(1,570 / 1,992)$ reported to be Christians, with $21 \%$ divided between traditional African beliefs, the Shembe religion - a combination of Christianity and Zulu culture - and other religions. Only two households reported to be of Muslim faith.

Thirty-eight percent $(761 / 1,992)$ of all of the households owned one or more dogs (range 1-19, median =2). Most of the dogs were either bought (44\%) or received as gifts (38\%). Individual descriptive statistics was attempted for every dog over suckling age; however, some records were incomplete. Data were collected on 1,667 individual dogs in 761 households. Ninety-nine percent $(1,650 / 1,667)$ of the dogs were at home during the time of interviews.

\section{Accessibility of owned dogs}

Seventy-two percent of the dogs $(1,147 / 1,598)$ were reported as owned by the head of the household. Values were missing 
for 69 responses (4\%). Children were responsible for $7 \%$ of the dogs, with $3 \%$ of dogs being cared for by everyone in the household. Other adult males or females owned the remaining $18 \%$. Sixty-five percent of dogs were reported as being able to be handled by everyone in the household with another $11 \%$, which could be easily handled by the children. Twenty-two percent could only be handled by the owner, and $<2 \%$ were reported as unmanageable. None of the dogs were identified as unowned or community-supported.

\section{Owner knowledge and valuation of rabies vaccine}

Eighty-six percent of survey respondents $(1,716 / 1,992)$ said they had heard of rabies. Nondog owners were 1.6 times more likely to have heard of rabies than dog owners. Seventy percent of vaccinated dogs had owners who were aware of rabies. Although rabies vaccination for dogs and cats can be obtained free of charge from GVS, respondents were asked to place a value in South African Rand on rabies vaccine for their animals. Owner valuation was measured by response to three cost categories for canine rabies vaccine and is reflected in Figure 2. Dog owners were also asked how far they would be willing to travel in order for their dogs to receive vaccinations. Ninety-one percent of dog owning respondents (693/761) said they would not travel further than a kilometer with their dogs in order to obtain vaccination.

\section{Rabies vaccination in owned dogs}

The individual dog survey allowed for the collection of the rabies vaccination status of each dog over suckling age in the household; however, rabies data points were missing for 47 of the 1,667 animals. Eighty-four percent $(1,361 / 1,620)$ of dogs with complete records had received a rabies vaccine at some point in their lifetime, and almost all of these dogs had last been vaccinated by an animal health technician during a rabies campaign. Less than $2 \%$ of dog owners reported that their dog had been vaccinated by a private veterinarian. Sixty-four percent $(1,043 / 1,620)$ of the dogs were reported as having been immunized within the last 1 year. Owners could provide vaccination cards in $82 \%$ of the cases.

Households that reported failure to attend the government campaigns were asked reasons. Without exception, the reasons were either lack of availability of the dog to vaccinators in the form of the owner or dog not being at the house or lack of awareness of the campaign (Table 1).

Seventeen percent of households surveyed $(337 / 1,992)$ owned cats with an average of 1.6 cats per household (range of $0-10)$. Sixty-three percent (342/542) of the owned cats were reported as vaccinated against rabies. Households with dogs were twice as likely to own cats as nondog owning households and $66 \%$ of rabies vaccinated cats came from homes where the dogs had been vaccinated in the most recent government campaigns. The ratio of dogs to cats in KZN surveyed households was 3.3:1.

\section{Nonvaccination risk regression model}

Our study sought measurable variables that influenced the vaccination status of owned dogs in the province with the purpose of sharing results with GVS in order to address these factors and increase the number of dogs vaccinated during future campaigns.

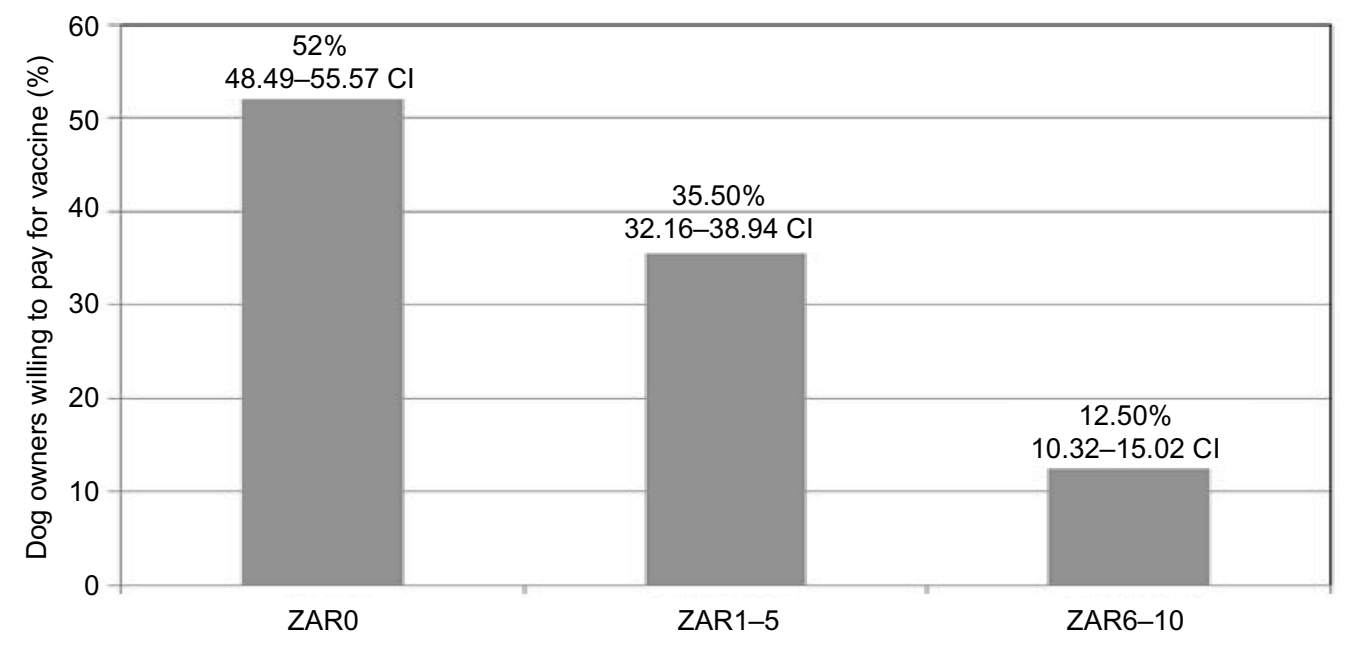

Figure 2 Owner valuation in ZAR of rabies vaccine.

Notes: I ZAR $=0.13$ USD. Totals are by percentage across all surveyed areas in KZN province, September 2009-January 201 I.

Abbreviations: $\mathrm{Cl}$, confidence interval; KZN, KwaZulu-Natal; USD, US Dollar; ZAR, South African Rand. 
Table I Reasons provided by KZN dog owners why they had not attended recent vaccination campaigns between September 2009 and January $201 \mathrm{I}(\mathrm{n}=222)$

\begin{tabular}{ll}
\hline Categories & Count \\
\hline Away from home & 80 \\
Did not know about campaign & 52 \\
Did not want vaccine & 5 \\
Too far to travel & 4 \\
Other reasons & 81 \\
Dog too young & \\
Dog ran away & \\
New dog to household & \\
\hline
\end{tabular}

Abbreviation: KZN, KwaZulu-Natal.

Risk of nonvaccination was measured at the dog level rather than the household level to account for households with multiple dogs. All of the terms found to be significant in the univariate analysis were entered into the model to determine factors associated with risk of nonvaccination. Area rather than area type was entered into the model as an independent variable so that each community surveyed would stand as its own category of interest. Although insignificant in univariate testing, the sex of the dog was forced into the model because it was a variable of interest. Constructs previously described in the "Methods" section were developed to combine similar significant variables of interest and were labeled as dog, household, and husbandry constructs. The final logistic regression model included terms for age of dog, area surveyed, household, dog, and husbandry constructs (Table 2). Eight percent of cases $(131 / 1,620)$ were deleted due to missing values for the response or explanatory variables. The sex of the $\operatorname{dog}(P=0.6572)$, religion $(P=0.1417)$, and owner of the $\operatorname{dog}(P=0.0648)$ were dropped from the model because each was found to be insignificant in the analysis of effects in eligibility for entry into the model $(P=0.05)$. The percent concordant was $72 \%$, reflecting that the model predicts the dependent variable correctly, and Somers' $D=0.446$. Among the study dogs, those dogs $<1$ year of age, dogs from the urban area of Umlazi, those with a low household construct score, a medium dog construct score, and a low husbandry construct score were most at risk of nonvaccination.

\section{Discussion}

Response rates $(92 \%-100 \%)$ were not adversely affected by conducting the surveys from 9 am to 3 pm when many people might be away from the household at work or school. Almost all owned dogs were at home during the time of the surveys, which is the same timeframe during which GVS would conduct rabies vaccination campaigns. As accessibility of dogs is considered one of the constraints to accomplishing
Table 2 Stepwise logistic regression model for risk of nonvaccination against rabies in owned dogs $(n=I, 489)$ in $K Z N$, South Africa, September 2009-January 2011

\begin{tabular}{|c|c|c|c|c|c|}
\hline Variable & $b$ & SE & $P$-value & OR & $95 \% \mathrm{Cl}$ \\
\hline Intercept & -0.9957 & 0.092 & $<0.0001$ & & \\
\hline \multicolumn{6}{|l|}{ Age (years) } \\
\hline$<1$ & 1.1562 & 0.1300 & $<0.0001$ & 5.605 & $3.607-8.709$ \\
\hline $\mathrm{I}-2$ & 0.2595 & 0.1116 & 0.0201 & 0.408 & $0.288-0.578$ \\
\hline 3 & -0.3645 & 0.1344 & 0.0067 & 0.219 & $0.146-0.327$ \\
\hline 4 & -0.4838 & 0.1716 & 0.0048 & 0.194 & $0.12-0.314$ \\
\hline$>5$ & -0.5674 & 0.1530 & 0.0002 & 0.178 & $0.115-0.277$ \\
\hline \multicolumn{6}{|l|}{ Area } \\
\hline Ixopo & -0.4005 & 0.1353 & 0.0031 & 0.288 & $0.159-0.523$ \\
\hline Pongola & 0.2647 & 0.1395 & 0.0577 & $0.56 \mathrm{I}$ & $0.309-1.017$ \\
\hline St Chad's & -0.4645 & 0.1437 & 0.0012 & 0.27 & $0.147-0.497$ \\
\hline Umlazi & 0.8435 & 0.2404 & 0.0005 & 2.675 & $1.378-5.120$ \\
\hline Esikhawini & -0.1096 & 0.2790 & 0.6944 & 0.386 & $0.168-0.886$ \\
\hline Wembezi & -0.1336 & 0.1796 & 0.4569 & 0.376 & $0.195-0.725$ \\
\hline \multicolumn{6}{|l|}{ Household } \\
\hline Low & 0.4316 & 0.0992 & $<0.0001$ & 2.15 & $1.508-3.065$ \\
\hline Medium & -0.0979 & 0.0929 & 0.2921 & 1.266 & $0.906-1.769$ \\
\hline High & -0.3337 & 0.1041 & 0.0014 & 0.790 & $0.565-1.104$ \\
\hline \multicolumn{6}{|l|}{ Dog } \\
\hline Low & 0.1297 & 0.1076 & 0.2280 & 1.656 & $1.165-2.355$ \\
\hline Medium & $0.245 \mathrm{I}$ & 0.0900 & 0.0065 & 1.859 & $1.392-2.484$ \\
\hline High & -0.3337 & 0.0927 & $<0.0001$ & 0.538 & $0.403-0.719$ \\
\hline \multicolumn{6}{|l|}{ Husbandry } \\
\hline Low & 0.2078 & 0.0900 & 0.0209 & 1.73 & $1.281-2.335$ \\
\hline Medium & 0.1329 & 0.1007 & 0.1892 & 1.604 & I. $145-2.247$ \\
\hline High & -0.3401 & 0.0933 & 0.0003 & 0.624 & $0.445-0.874$ \\
\hline
\end{tabular}

Note: Effects model pivots around 0.

Abbreviations: $\mathrm{Cl}$, confidence interval; KZN, KwaZulu-Natal; OR, odds ratio; SE, standard error.

the recommended $70 \%$ vaccination coverage, it should be considered that this value or higher is achievable in KZN. An existing vaccination status was reported for $84 \%$ of owned dogs in the areas included in this study - with $64.24 \%$ of these having been vaccinated within a 12 -month period prior to the survey. This number is in close agreement with a study performed in Bohol, Philippines, where 64\% of dog owners had their dogs vaccinated within a year prior to survey and is slightly higher than the $56 \%$ reported from the Eastern Cape province. $^{13,22}$ Of the surveyed dog population in KZN, 253 $(16 \%)$ had never received a rabies vaccine.

Eighty-two percent of dog owners said that they had vaccination cards from the last immunization event attended, but bias is possible in the response to the proof of vaccination query as the cards do not specifically identify individual animals. In other developing countries, $40 \%-82 \%$ of $\operatorname{dog}$ owners could provide some form of proof their dogs had been vaccinated. ${ }^{22-24}$ When the vaccinated population is only considered for those cases where owners could produce cards, 
then the coverage results in $69 \%$ of surveyed dogs having been vaccinated previously in their lifetime and $53 \%$ of dogs being vaccinated within the last year. Because this study only concerned owned dogs, vaccination coverage estimates presented here cannot be considered for the entire target dog population of KZN as estimates do not include the ownerless or stray dog population. However, it is a commonly held concept that the ownerless or feral dog population in Africa is low and no dogs were reported as unowned or stray during the interviews. ${ }^{25,26}$

Most of the surveyed households are utilizing the free government rabies vaccine services as $<2 \%$ of dog owners reported the use of a private veterinary clinic. Private veterinarians were also underutilized in Bohol ${ }^{23}$ and Tunisia, ${ }^{22}$ where only $3 \%$ and $10 \%$ of dogs were reported to have been vaccinated at a veterinary clinic.

In Africa, factors that have been shown to affect dog accessibility are household settlement patterns, religion, culture, and animal husbandry practices. ${ }^{1,5,17,27}$ Neither culture nor religion played a significant role in the vaccination status of the surveyed population. Reasons provided by respondents for why owners did not bring their dogs to the government vaccinations included owner unawareness of the event, owner was away from the household at the time of the campaigns or they considered the distance too far to bring the dog for vaccine (61\%), dog ran away from technicians, the dog was new to the household, or owner thought the dog was too young $(36 \%)$. None of the owners reported not presenting their dogs because they could not or would not handle the dogs. In N'Djaména, Chad, dog owners not attending vaccination points reported not being able to handle their animals (25\%), being unaware of the campaign or lack of time to attend (19\%), and dogs being too young or running away $(17 \%) .^{28}$ In this study, $11 \%$ of dogs were reported as only being able to be handled by the children of the household, and $65 \%$ could be handled by everyone in the household. Eighty-seven percent of the dog owning population surveyed were willing to pay less than South African Rand 5 or US $\$ 0.65$ for rabies vaccination.

The model generated for our study shows that a risk factor for nonvaccination against rabies is age. Dogs $<1$ year of age were at much greater risk than dogs aged 3 years or older. In Santa Cruz de la Sierra, Bolivia, researchers found dogs 1-11 months of age most at risk of nonvaccination against rabies. ${ }^{15}$ Similar results have been reported from Mexicali, Mexico, Guayaquil, Ecuador, and Bohol, Philippines, for dogs aged $<1$ year., ${ }^{9,1422}$ Specific reasons were not sought as to why this young age group of dogs in KZN was not vaccinated; however, it can be considered based upon dog owners' comments that the dogs arrived at the household after the most recent campaigns or that the owners considered the dog too young for vaccination at the time. The thought pattern of dogs being too young for vaccination is of concern as $52 \%$ of rabies cases were reported as occurring in dogs aged 3-11 months in Guayaquil. ${ }^{14}$ Owner education toward targeting this younger age group of dogs should be part of the rabies prevention program. These younger dogs are typically accessible since they are less mobile, not usually wary of people, and are frequently found playing with younger children. ${ }^{29}$ According to Chappuis, ${ }^{30}$ young puppies that had maternally derived antibodies to rabies could still mount an immune response when vaccinated with high quality commercially available rabies vaccines - such as is being used in KZN. In contrast, age was not a significant factor of nonvaccination in a report from Nigeria. ${ }^{10}$

The model showed that dogs from urban Umlazi were most at risk of not being vaccinated. The reasons for dogs in this area being at greater risk of nonvaccination may be related to the fact that Umlazi is a busy urban township with a large working community where dog owners may be away from home during the campaign events. Perry et $\mathrm{al}^{3}$ recommended high human population density suburbs should be priority areas for achieving high levels of vaccine coverage due to the effective contacts possible in dense dog populations of free roaming dogs. As previously stated, conducting campaigns with extended hours, on Saturdays, or during school holidays may be advantageous in this community setting.

Dogs in the lowest household construct were most at risk of nonvaccination compared to the medium and higher constructs. Persons who are unaware of rabies and own fewer animal might have less motivation to seek preventative health care for their dogs, whereas Cleaveland et $\mathrm{a}^{31}$ refer to fear of dog rabies in some Tanzania communities being a larger concern than malaria, which is a disease of higher prevalence. Dogs with owners who were unaware of the zoonotic implication of rabies were significantly less likely to have vaccinated dogs than those owners who knew of rabies in Ibadan. ${ }^{10}$ Dogs in the lowest husbandry construct were found to be most at risk of nonvaccination against rabies. This result indicates that lower standard animal husbandry practices in developing countries are a point to be addressed in educational aspects of rabies program development. Sixty-three percent of owned dogs in KZN were reported to be unrestrained at all times and free to roam at will. Interestingly, free roaming dogs in Nigeria were more likely to be vaccinated against rabies than confined dogs, as owners who restrain their dogs feel they 
are less likely to contact rabies infected dogs. ${ }^{10}$ The model showed dogs in the medium dog construct were more at risk of not being vaccinated against rabies than the lower or higher level dog construct. The dog construct consisted of the variables for the source of the dog and purpose of the dog. Dogs were found to be guard dogs in $87 \%$ of households. The two main sources from where owners obtained dogs were as gifts (38\%) and purchases (44\%). Dogs in Mexicali, Mexico, that were obtained as a gift, found on the street, or came from the owner's bitch were found to be twice the risk of nonvaccination as dogs that were purchased. ${ }^{9}$ Although the source of the dog was not a significant factor in Nigeria, the price paid for the dog was a significant factor in risk of nonvaccination against rabies. ${ }^{10}$ Dog owners in this study reported they were more likely to present their dogs for vaccination when the vaccines were free of charge (52\%) and less than a kilometer from their homes (91\%). Dog owners in Nigeria and Eastern Cape province were also dependent upon home vaccine delivery. ${ }^{10,13}$

While the model cannot predict the probability of a certain dog being vaccinated, it pointed out factors that could more heavily influence the risk of nonvaccination. As one goal of the study was to elucidate factors that could influence the vaccination status of owned dogs in KZN, this model revealed that age of the dog and area of residence are two major factors that should be considered in rabies program planning for this province. The overall age and sex structure of the owned dog population are comparable to that found in other developing African countries, which could indicate a high population turnover also placing the population at risk of nonvaccination. ${ }^{6,32}$

\section{Conclusion}

The study indicates that $64 \%$ of the owned dog population of KZN are reported to have been rabies vaccinated within the year prior to the survey using a vaccine that is labeled with a 3-year duration of immunity. The willingness for owners to pay for rabies vaccine is low and might be a reflection of the habituation to the provision of free immunization by GVS. Door-to-door vaccine delivery has been shown to be preferred not only in this province but in other parts of southern Africa as well. Marketing of campaigns and rabies awareness are important motivators for owners to present their animals for vaccination. The study revealed that age of the dog and area of residence are two major risk factors for nonvaccination that should be considered in rabies program planning for this province. Other factors explaining why canine rabies remains endemic to KZN might be related to the high population turnover, high number of free roaming dogs, and inadequate vaccination coverage in high risk areas. With the low vaccination coverage estimated through this study for all sexes and age groups, it should be considered that many young puppies found during vaccination campaigns are naïve, and thus should be vaccinated during these opportunities.

\section{Acknowledgments}

The authors would like to acknowledge the Managing Veterinarians of the KwaZulu-Natal Department of Agriculture, Environment and Rural Development Dr Dumasani Mtshali and Dr Temba Sikhakhane; the KZNDAERD Epidemiology staff Dr Keith Perrett, Mr Kevin le Roux, Mrs Tokkie Tischendorf, Mrs Debbie Cooke, and Ms Vigie Govendor; and State Veterinarians and Control animal health technicians Ms Joey Peens, Mr Amos Mateta, Mr Carel Burger, Mr Hadebe, Dr Mbaga Lukubisa, Dr Shavetha Dhanilall, Dr Johan Blignaut, Dr Alan Rowe, and Dr Rob Paterson for providing equipment and personnel which aided in the completion of this study. We would also like to express sincere appreciation to Dr Frans Kanfer and Mrs Jaqui Sommerville of the statistics department at the University of Pretoria, Hatfield campus, for assistance with regression modeling procedures.

\section{Disclosure}

The authors report no conflicts of interest in this work.

\section{References}

1. WHO. Guidelines for Dog Rabies Control. Geneva, Switzerland: World Health Organization; 1987.

2. Coleman PG, Dye C. Immunization coverage required to prevent outbreaks of dog rabies. Vaccine. 1996;14(3):185-186.

3. Perry BD, Kyendo TM, Mbugua SW, Price JE, Varma S. Increasing rabies vaccination coverage in urban dog populations of high human population density suburbs: a case study in Nairobi, Kenya. Prev Vet Med. 1995;22(1-2):137-142.

4. Bishop GC, Durrheim DN, Kloeck PE, Godlonton JD, Bingham J, Speare R. Rabies Advisory Group: Rabies: Guide for the Medical, Veterinary and Allied Professions. 2nd ed. Pretoria: Government Printer; 2010.

5. Kaare M, Lembo T, Hampson K, et al. Rabies control in rural Africa: evaluating strategies for effective domestic dog vaccination. Vaccine. 2009;27(1):152-160.

6. Hergert M. Characteristics of High Risk Dog Populations in Rabies Endemic KwaZulu-Natal Province South Africa [dissertation]. Pretoria: University of Pretoria; 2013.

7. Cleaveland S, Kaare M, Tiringa P, Mlengeya T, Barrat J. A dog rabies vaccination campaign in rural Africa: Impact on the incidence of dog rabies and human dog-bite injuries. Vaccine. 2003;21(17-18):1965-1973.

8. Knobel DL, Cleaveland S, Coleman PG, et al. Re-evaluating the burden of rabies in Africa and Asia. Bull World Health Organ. 2005;83:360-368. 
9. Flores-Ibarra M, Estrella-Valenzuela G. Canine ecology and socioeconomic factors associated with dogs unvaccinated against rabies in a Mexican city across the US-Mexican border. Prev Vet Med. 2004; 62(2):78-87.

10. Awoyomi OJ, Adeyemi IG, Awoyomi FSO. Socioeconomic factors associated with non-vaccination of dogs against rabies in Ibadan, Nigeria. Nig Vet J. 2007;28(3):59-63.

11. Matter HC, Wandeler AI, Neueschwander BE, Harischandra LPA, Meslin FX. Study of the dog population and the rabies control activities in the Mirigama area of Sri Lanka. Acta Trop. 2000;75:95-108.

12. Dürr S, Meltzer MI, Mindekem R, Zinsstag J. Owner valuation of rabies vaccination of dogs, Chad. Emerg Infect Diseases. 2008; 14(10):1650-1652.

13. Van Sittert SJ, Raath J, Akol GW, Miyen JM, Mlahlwa B, Sabeta CT. Rabies in the Eastern Cape province of South Africa - where are we going wrong? J S Afri Vet Assoc. 2010;81(4):207-215.

14. Beran GW. Urban rabies. In: Baer GM, editor. The Natural History of Rabies. 2nd ed. Boca Raton, FL: CRC Press; 1991:427-443.

15. Suzuki K, Pereira JAC, Frías LA, López R, Mutinelli LE, Pons ER. Rabies-vaccination coverage and profiles of the owned-dog population in Santa Cruz de la Sierra, Bolivia. Zoonoses Public Health. 2008; 55(4):177-183.

16. Nel LH, Markotter W. Lyssaviruses. Crit Rev Microbiol. 2007;33(4): 301-324.

17. Perry BD. Dog ecology in eastern and southern Africa: Implications for rabies control. Onderstepoort J Vet Res. 1993;60(4):429-436.

18. Garson GD: Sampling. [Blue book series]. Statistical Associates Publishing; 2009 [30 pages]. Available from: http://www.statisticalassociates. com/sampling.pdf. Accessed May 2009.

19. Random.org [homepage on the Internet]. Dublin: Randomness and Integrity Services Ltd. Available from: http://www.random.org. Accessed August 2009-February 2011.

20. WHO. Report of Dog Ecology Studies Related to Rabies. Geneva: World Health Organization; 1988.

21. Hosmer DW, Lemeshow S. Applied Logistic Regression. New York: John Wiley \& Sons; 2000.
22. Davlin S, Lapiz SM, Miranda ME, Murray K. Factors associated with dog rabies vaccination in Bohol, Philippines: results of a cross-sectional cluster survey conducted following the island-wide rabies elimination campaign. Zoonoses Public Health. 2013;60:494-503.

23. Touihri L, Zaouia I, Elhili K, Dellagi K, Bahloul C. Evaluation of mass vaccination campaign coverage against rabies in dogs in Tunisia. Zoonoses Public Health. 2011;58(17):110-118.

24. Robinson LE, Miranda ME, Miranda NL, Childs JE. Evaluation of a canine rabies vaccination campaign and characterization of owned-dog populations in the Philippines. Southeast Asian J Trop Med Public Health. 1996;27:250-256.

25. Gsell AS, Knobel DL, Kazwala RR, Vounatsou P, Zinsstag J. Domestic dog demographic structure and dynamics relevant to rabies control planning in urban areas in Africa: the case of Iringa, Tanzania. BMC Vet Res. 2012;8:236-246.

26. Jibat T, Hogeveen H, Mourits MCM. Review on dog rabies vaccination coverage in Africa: A question of dog accessibility or cost recovery? PLOS Negl Trop Dis. 2015;9(2):e0003447.

27. Matter HC, Daniels TJ. Dog ecology and population biology. In: Macpherson CNL, Meslin FX, Wandeler AI, editors. Dogs, Zoonosis and Public Health. New York: CABI Publishing; 2000:17-62.

28. Kayali U, Mindekem R, Yémadji N, et al. Coverage of pilot parenteral vaccination campaign against canine rabies in N'Djaména, Chad. Bull World Health Organ. 2003;81:739-744.

29. Barrat J, Blasco E, Lambot M, et al. Is it possible to vaccinate young canids against rabies and to protect them? In: Proceedings of the Sixth Southern and Eastern African Rabies Group meeting; June 18-21, 2001; Lilongwe.

30. Chappuis G. Neonatal immunity and immunization in early age: Lessons from veterinary medicine. Vaccine. 1998;16(14-15):1468-1472.

31. Cleaveland S, Kaare M, Knobel D, Laurenson MK. Canine vaccination - providing broader benefits for disease control. Vet Microbiol. 2006;117(1):43-50.

32. Kitala PM, McDermott JJ, Kyule MN, Gathuma JM, Perry B, Wandeler AI Dog ecology and demography information to support the planning of rabies control in Machakos District, Kenya. Acta Trop. 2001;78: $217-230$.
Veterinary Medicine: Research and Reports

\section{Publish your work in this journal}

Veterinary Medicine: Research and Reports is an international, peer-reviewed, open access journal publishing original research, case reports, editorials, reviews and commentaries on all areas of veterinary medicine. The manuscript management system is completely online and includes a very quick and fair peer-review system.

\section{Dovepress}

Visit http://www.dovepress.com/testimonials.php to read real quotes from published authors.

Submit your manuscript here: http://www.dovepress.com/veterinary-medicine-research-and-reports-journal 